PlastOx 2007 (2009) 205-219

(C) EDP Sciences, 2009

DOI: $10.1051 /$ ptox $/ 2009015$

\title{
Détection et quantification des phénomènes de fissuration assistée par l'environnement
}

\section{Fregonese}

INSA-Lyon - MATEIS-RI 2 S, CNRS UMR 5510, 69621 Villeurbanne Cedex, France

e-mail : marion.fregonese@insa-lyon.fr

\begin{abstract}
Résumé. Ce cours a pour but de décrire les techniques et démarches expérimentales permettant la détection et la quantification des phénomènes de fissuration assistée par l'environnement. A partir d'exemples récents tirés de la littérature, nous montrons en premier lieu qu'une analyse métallographique et/ou fractographique peut permettre d'obtenir des résultats intéressants. Pour des informations quantitatives sur le développement des fissures, les potentialités et complémentarités des techniques d'émission acoustique et de suivi électrique sont évaluées. Ces deux techniques, tout comme les méthodes électrochimiques stationnaires et non stationnaires et la microtomographie X, qui sont également décrites, offrent en outre la possibilité d'un suivi in-situ de la propagation de l'endommagement. Nous présentons enfin une revue critique des méthodes, principalement non destructives, appliquées sur site industriel pour une estimation prédictive de la durée de vie des structures.
\end{abstract}

\section{INTRODUCTION}

La compréhension, la modélisation et la prédiction des processus de fissuration assistée par l'environnement (Corrosion Sous Contrainte, Fatigue Corrosion, Fragilisation Par l'Hydrogène) ne sont possibles que si l'on est capable de détecter l'endommagement et de le quantifier, c'est-à-dire de :

- savoir quand il s'amorce;

- savoir où il s'amorce;

- déterminer son (ses) mode(s) de propagation;

- déterminer ses interactions avec la microstructure.

Il est alors possible d'évaluer le rôle respectif des différents paramètres chimiques, mécaniques, métallurgiques mis en jeu, étape nécessaire à l'établissement de modèles phénoménologiques, et de quantifier l'influence de ces paramètres afin de les prendre en compte dans une modélisation micro-mécanique.

Cette détection et cette quantification passent par la mise en place de techniques et de démarches expérimentales que nous nous proposons de décrire ici.

Nous verrons en premier lieu qu' une analyse métallographique et/ou fractographique «simple » peut permettre d'obtenir des résultats intéressants. Les méthodes permettant d'avoir accès à des informations quantitatives, in situ, sur le développement des fissures seront ensuite décrites. Nous verrons pour finir quelles méthodes, principalement non destructives, sont appliquées sur site industriel pour une estimation prédictive de la durée de vie des structures.

\section{QUANTIFICATION DES CINÉTIQUES DE FISSURATION PAR ANALYSE MÉTALLOGRAPHIQUE ET FRACTOGRAPHIQUE}

Une «simple» analyse métallographique et/ou fractographique peut permettre de proposer une quantification des cinétiques d'endommagement par Corrosion Sous Contrainte, obtenu lors d'essais mécaniques divers (traction lente à vitesse de déformation imposée, charge constante, flexion...) réalisés en présence d'un milieu agressif. Nous illustrerons nos propos par l'exemple du modèle morphologique proposé par G. Santarini et al. [1, 2], et nous préciserons les apports et les limites de l'analyse fractographique. 


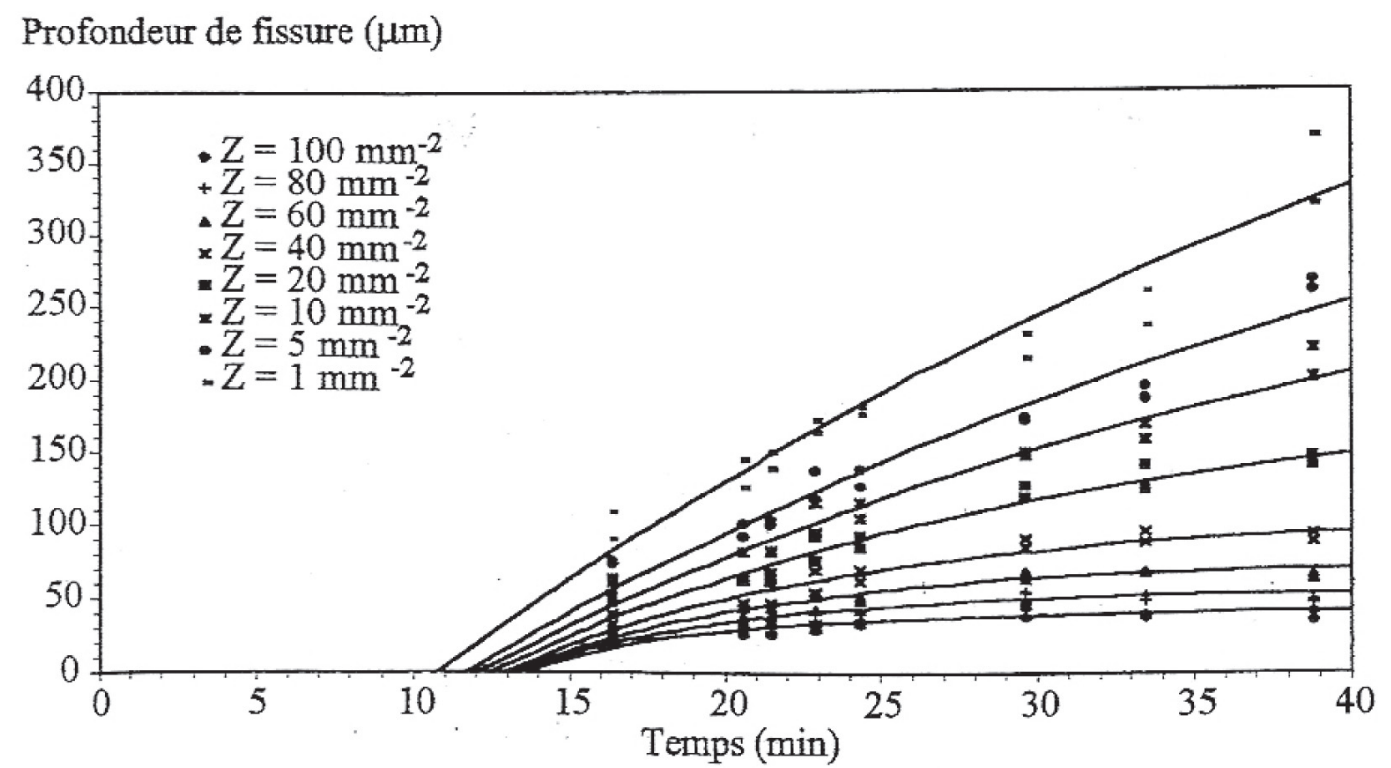

Figure 1. Évolution de la profondeur des fissures en fonction de leur densité surfacique (acier $\mathrm{Z} 2 \mathrm{CN} 18.10, \mathrm{MgCl}_{2}$, $\left.153^{\circ} \mathrm{C}\right)[1]$.

\subsection{Modélisation du développement de fissures de CSC par leur analyse morphologique}

Cette démarche a été mise en œuvre suite à la constatation que, dans de nombreux cas, la quantification des cinétiques d'endommagement par Corrosion Sous contrainte était entreprise soit par une approche purement théorique, soit par une approche purement empirique, mise en place dans des conditions expérimentales souvent éloignées des cas réels, soit par une démarche intermédiaire, semi-empirique, faisant appel à des hypothèses mécanistiques et n'étant donc pas généralisable à divers couples matériau/milieu [3]. La démarche mise en place a donc consisté à proposer une méthode de caractérisation complète et de prédiction basée sur une simple analyse de la morphologie des fissures, à savoir la répartition de leur taille et de leur forme. Les paramètres d'étude sont alors principalement la densité $\mathrm{Z}$ des fissures de profondeur supérieure à $\mathrm{l}$ à l'instant $\mathrm{t}$, ainsi que la profondeur de la trace des fissures observées sur un plan de coupe parallèle à la contrainte maximale de traction. Ces données sont obtenues par polissages successifs de la surface des éprouvettes, ayant subi des tests de traction lente interrompus à différentes durées d'essai.

Ainsi, pour le système acier inoxydable austénitique / chlorure de magnésium bouillant, les résultats suivants ont été obtenus [1] :

- les fissures se propagent différemment selon leur taille (fig. 1) : la vitesse des fissures décroit au cours de l'essai et cette décroissance est une fonction croissante de la densité $\mathrm{Z}$ de fissures plus profondes que la fissure considérée ; ce résultat a été expliqué par «l'effet d'écran» d'origine purement mécanique qu'exercent les fissures de grande taille sur les plus petites : les fissures amorcées les premières «atrophient» les fissures qui apparaissent ensuite (il en découle une diminution de leur vitesse initiale) et ne leur permettent pas d'atteindre de grandes profondeurs ;

- un phénomène de saturation de l'amorçage apparent a été observé ; il est la conséquence de l'effet d'écran exercé par les fissures de grande taille ;

- cet effet d'écran est accentué par une diminution de la vitesse d'allongement relatif.

Le même type d'approche a été conduit pour le système alliage 600 / milieu primaire REP [2] :

- le même effet d'écran a été observé ; 


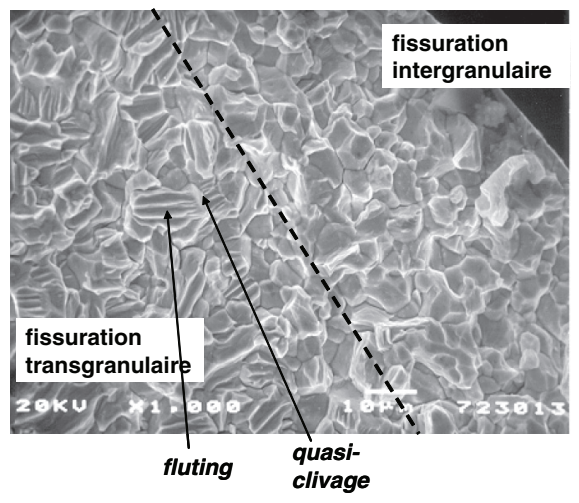

Figure 2. Propagation de fissures intergranulaires puis transgranulaires (Zircaloy-4 irradié, méthanol iodé, $\left.25^{\circ} \mathrm{C}\right)[6]$.

- une première phase de propagation lente des fissures est caractérisée par une vitesse dépendant de la vitesse d'allongement relatif et de Z ; les fissures les plus courtes ne dépassent pas ce premier stade de propagation ;

- les fissures les plus longues voient leur vitesse de propagation augmenter au cours de l'essai, soit du fait de la disparition des interactions entre petites et grandes fissures, soit parce qu'un facteur d'intensité de contrainte critique en fond de fissure est alors atteint.

Dans les deux cas, la démarche a mis en évidence l'importance du paramètre vitesse de déformation locale en pointe de fissure. Elle doit être désormais validée par une approche mécaniste des phénomènes.

Notons que si cette démarche expérimentale est apparue comme novatrice lors de son développement, en particulier du fait de sa simplicité, elle a nécessité une caractérisation lourde et fastidieuse des échantillons. Le développement actuel de nouvelles techniques de caractérisation de l'état de fissuration d'échantillons massifs par microtomographie X par exemple pourrait lui donner un nouvel essor.

\subsection{Analyse fractographique}

Les informations contenues sur un faciès de rupture sont très riches, puisqu'on peut, par visualisation directe au Microscope Electronique à Balayage, avoir accès aux modes de fissuration (inter, transgranulaire, clivage (fig. 2) ... ), et mesurer des tailles de fissures. L'analyse fractographique permet de retracer en quelque sorte l'avancée de la fissure de CSC.

Cette analyse a été largement utilisée comme base expérimentale du développement de modèles phénoménologiques de Corrosion Sous Contrainte. On peut par exemple citer les travaux de Paret [4], qui confirment en partie les prédictions du «Corrosion Enhanced Plasticity Model».

Elle peut permettre également de quantifier des cinétiques d'endommagement. Ainsi, des vitesses de propagation ont été évaluées par relevé des profondeurs maximales de fissures sur des faciès de rupture d'éprouvettes en acier inoxydable austénitique 316L hypertrempé sollicité en traction lente sous polarisation cathodique en milieu $\mathrm{H}_{2} \mathrm{SO}_{4}$ [5]. Il a ainsi été démontré que les vitesses de propagation de fissures étaient croissantes avec la vitesse de déformation appliquée et donc qu'un phénomène de transport de l'hydrogène par les dislocations était à l'origine de la propagation de l'endommagement.

Cette démarche nécessite cependant d'être capable d'estimer le temps d'amorçage des fissures, qu'il faut déduire de la durée de l'essai pour obtenir un temps de propagation. Cette détermination peut se faire expérimentalement par un suivi électrochimique du potentiel de corrosion de l'échantillon ou par Emission Acoustique, méthodes que nous développerons par la suite. Une autre possibilité est de tester 
des éprouvettes pré-entaillées ou pré-fissurées, permettant de s'affranchir alors de l'étape d'amorçage des fissures.

Notons que l'analyse fractographique ne contraint pas forcément à conduire les essais de CSC jusqu'à rupture. L'essai mécanique peut être interrompu, puis l'éprouvette être cassée ultérieurement, par exemple en présence d'azote liquide, ce qui permet de figer l'endommagement. Un chauffage à l'air de l'éprouvette avant rupture peut permettre en outre une oxydation légère de la zone fissurée et faciliter son repérage sur le faciès.

\section{MÉTHODES DE SUIVI IN SITU DU DÉVELOPPEMENT DE FISSURES DE CORROSION SOUS CONTRAINTE}

Les enjeux d'une détection in-situ de l'endommagement sont multiples. Cette détection peut aider à la réalisation de la démarche expérimentale, en permettant par exemple l'interruption d'un essai au cours d'une phase donnée de la propagation de l'endommagement ; elle peut au contraire permettre de recueillir des informations sur toutes les phases de développement de l'endommagement en un seul essai ; elle peut enfin permettre de s'affranchir d'un contrôle «destructif» de l'échantillon après essai (l'essai de CSC étant lui-même destructif !).

Nous nous proposons de décrire ici quatre techniques expérimentales permettant d'atteindre ces objectifs :

- les méthodes électrochimiques ;

- l'Emission Acoustique et le suivi électrique, qui présentent des caractères complémentaires ;

- la microtomographie X.

Nous préciserons le principe de ces techniques et illustrerons leur utilisation au travers d'exemples issus de la littérature.

\subsection{Les méthodes électrochimiques}

La nature électrochimique des processus de corrosion rend incontournable l'utilisation de méthodes électrochimiques pour les étudier, c'est-à-dire identifier les mécanismes à leur origine, les quantifier, voire les anticiper. Ces méthodes peuvent être stationnaires ou non, lorsqu'une perturbation est appliquée au système étudié. Elles permettent toutes en général de suivre son évolution au cours du temps.

\subsubsection{Méthodes stationnaires}

Le cas de la corrosion sous contrainte, comme tous les cas de corrosion localisée, est particulier, dans le sens où seule une zone peu étendue du métal est fragilisée, en regard de toute la surface sollicitée sur laquelle les mesures électrochimiques sont en général effectuées. Cette limitation peut toutefois désormais être contournée par l'utilisation de techniques électrochimiques locales, en plein essor actuellement.

Il faut bien séparer les mesures électrochimiques en temps réel, qui nous intéressent ici, des mesures réalisées dans le milieu réactionnel mais sans contrainte appliquée, telles les courbes potentiocinétiques ou la technique EPR, qui permettent de définir la sensibilité globale de l'alliage métallique testé vis-à-vis d'une fissuration résultant de la transition état actif - état passif en fond de fissure [7].

Les mesures électrochimiques en temps réel sont mises en œuvre à potentiel libre de manière à ce que les mesures n'influent pas sur le développement des fissures. Elles consistent en des mesures de fluctuations de courant et/ou de potentiel, rassemblées sous le terme de bruit électrochimique, et en l'établissement de relations entre ces fluctuations et les différentes étapes de la fissuration [7]. Le courant de corrosion est en général mesuré entre l'échantillon testé (électrode de travail) et une contre-électrode (en général un échantillon de même nature mais rendu insensible à la CSC), via un ampèremètre à 

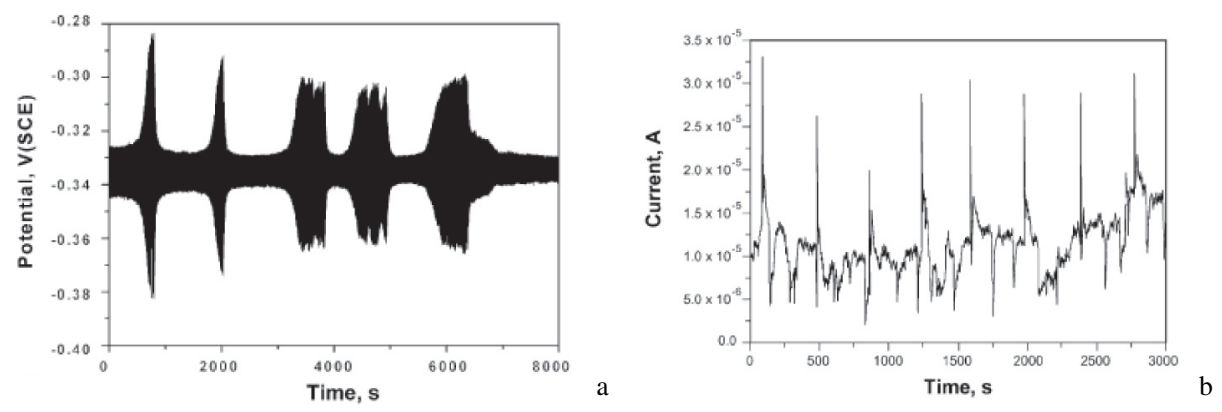

Figure 3. Fluctuations de potentiel (a) liées à l'amorçage des fissures et fluctuations de courant (b) liés aux phénomènes de dissolution anodique (acier $316, \mathrm{NaCl}+\mathrm{Na}_{2} \mathrm{SO}_{4}$ acidifié, $108^{\circ} \mathrm{C}$ ) [9].

résistance nulle. Les fluctuations de potentiel sont mesurées quant à elles entre l'électrode de travail et une électrode de référence, via un voltmètre à haute impédance d'entrée. En théorie, toute modification électrochimique de la surface du métal peut être détectée par des variations de courant et de potentiel entre les électrodes couplées. Des traitements temporels et fréquentiels adaptés des signaux de bruit (déviation standard, RMS, résistance de bruit...) peuvent alors permettre de détecter l'amorçage des fissures de CSC [8].

Une telle démarche a par exemple été retenue pour étudier la CSC d'un acier inoxydable austénitique $316 \mathrm{~L}$ sollicité en flexion dans une solution bouillante de $\mathrm{NaCl}$ et $\mathrm{Na}_{2} \mathrm{SO}_{4}$ [9]. Après 5 heures d'essai, l'enregistrement de fluctuations de potentiel «en paquets » a montré qu'un temps de latence de 5 heures est nécessaire à l'amorçage des fissures de CSC (fig. 3a). Le temps total (17 h) durant lequel ces signaux sont enregistrés, renseigne sur le temps d'amorçage des fissures. Les fluctuations périodiques du courant enregistrées durant la suite de l'essai (fig. 3b) renseignent quant à elles sur la périodicité des phénomènes de dissolution (phase d'augmentation du transitoire de courant) et de repassivation (diminution du transitoire) inhérents à la propagation des fissures de CSC.

\subsubsection{Méthodes non stationnaires}

Bien que peu développées dans ce domaine, des mesures d'impédance électrochimique (méthode non stationnaire appelée Spectroscopie d'Impédance Electrochimique) sont également utilisées pour suivre les phénomènes de CSC. De façon générale, ces mesures consistent à étudier la réponse d'un système électrochimique suite à une perturbation qui est, le plus souvent, un signal alternatif de faible amplitude. Cette technique permet de différentier les phénomènes réactionnels par leur temps de relaxation : les processus rapides sont caractérisés à hautes fréquences ; lorsque la fréquence appliquée diminue, apparaît la contribution des étapes plus lentes, comme les phénomènes de transport ou de diffusion en solution.

En pratique, la mesure d'impédance consiste à surimposer, à un point de fonctionnement stationnaire, une perturbation sinusoïdale $\Delta \mathrm{E}=|\Delta \mathrm{E}| \cdot \exp (\mathrm{j} \omega t)$. Il en résulte alors un courant $\Delta \mathrm{I}=$ $|\Delta \mathrm{I}| \cdot \exp (\mathrm{j} \omega \mathrm{t}+\phi)$, qui se superpose au courant stationnaire, $\phi$ correspondant au déphasage du courant alternatif par rapport au potentiel. Des travaux récents montrent les potentialités de cette technique pour la détection de la fissuration au cours d'essais de mise sous charge constante ou de traction lente réalisés à température ambiante ou à haute température en autoclave [10]. Le principe de la mesure consiste à suivre l'évolution du déphasage d'impédance entre un échantillon sollicité mécaniquement et un échantillon non contraint, tous deux placés dans le milieu corrosif. Les mesures sont réalisées quasisimultanément sur les deux échantillons. L'augmentation du déphasage est associée au développement des fissures de CSC. Une modélisation préliminaire de la surface contenant une ou plusieurs fissures a montré l'existence d'une fréquence optimale pour la détection de la fissuration qui dépend de la taille 

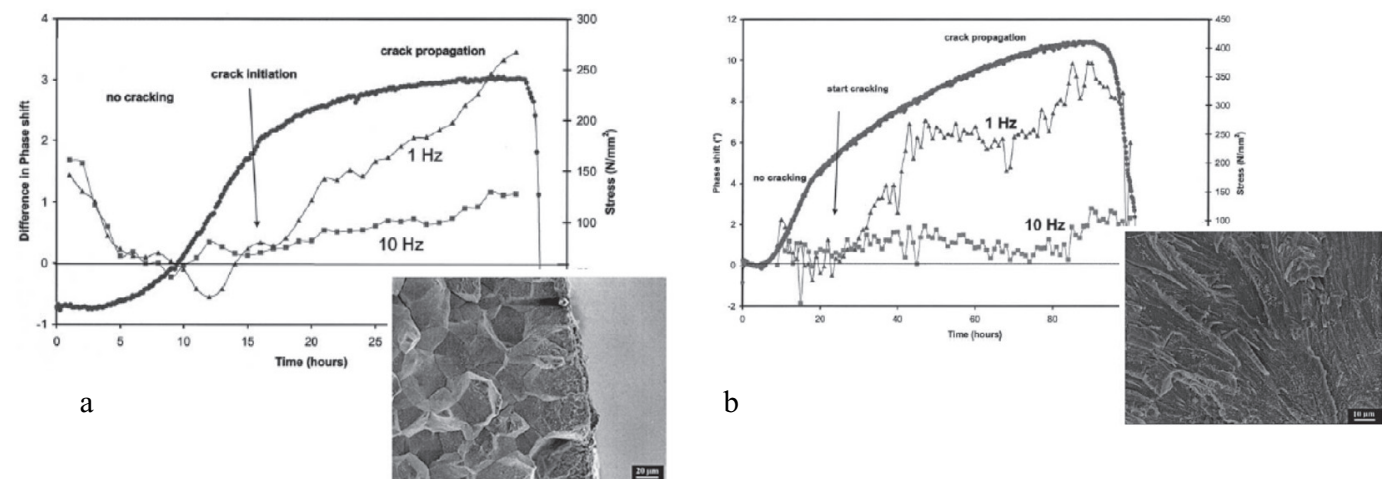

Figure 4. Détection de l'amorçage de fissures intergranulaires (a : acier 304 sensibilisé, $\mathrm{Na}_{2} \mathrm{SO}_{4}, 300^{\circ} \mathrm{C}$ ) et transgranulaires ( $\mathrm{b}$ : acier 304 hypertrempé, $\mathrm{Na}_{2} \mathrm{SO}_{4}, 300^{\circ} \mathrm{C}$ ) par mesure du déphasage d'impédance électrochimique [10].

des fissures. Ainsi, pour les petites fissures, la fréquence de détection optimale est plus élevée que pour les plus grosses fissures, ce qui contraint à utiliser une certaine gamme de fréquences de perturbation du système.

Avec cette méthode, la détection de fissures intergranulaires (fig. 4a) comme transgranulaires (fig. 4b) a été possible au cours d'un essai de traction lente.

\subsection{L'émission acoustique}

L'Emission Acoustique (EA) est la conséquence d'un phénomène de création d'ondes élastiques transitoires résultant de microdéplacements internes à un matériau et/ou à son environnement immédiat. Elle est souvent associée à l'évolution d'un défaut dans une pièce soumise à une contrainte. Si ce défaut évolue sous l'effet de la contrainte appliquée, il devient une source d'ondes élastiques transitoires qui se propagent au sein du matériau, jusqu'à sa surface, où elles sont détectées. Par conséquent, la détection de l'émission acoustique permet de révéler la présence de défauts évolutifs, en temps réel.

Il s'agit d'une méthode de contrôle non destructif (CND), non intrusive [11]. En général, la technique d'EA ne fournit pas d'information sur la taille du défaut se propageant, mais renseigne sur l'évolution de ce défaut et sur sa localisation au sein de la structure. Cette méthode volumique apporte ainsi, et c'est là son originalité, une information locale à partir d'une analyse globale de la pièce sollicitée.

Cette définition générale de l'émission acoustique doit être quelque peu modifiée dès lors que l'on s'intéresse à la détection de processus liés à la corrosion. En effet, le défaut, s'il doit toujours être en cours d'évolution pour être détecté, se trouve en général en surface du matériau lorsqu'il résulte d'un phénomène de corrosion, excepté dans le cas de la décohésion interne par l'hydrogène (HIC : Hydrogen Induced Cracking). De plus, son évolution n'est pas toujours uniquement liée à l'application d'une contrainte mécanique, mais aussi, souvent, à un processus électrochimique (dépassivation, réduction du proton ou de l'oxygène dissous).

De plus, une autre spécificité du suivi des phénomènes de corrosion par EA, en comparaison avec les endommagements de type mécanique, réside dans le fait que ce n'est pas, en général, l'évolution du défaut lui-même (dissolution, piqûre, crevasse) que l'on détecte (excepté le cas du développement d'une fissure), mais un processus annexe qui lui est associé (dégagement d'hydrogène, formation de produits de corrosion...). Ces différentes sources d'EA ont été répertoriées par Yuyama il y a une vingtaine d'années (fig. 5) [12]. On constate que nombre d'entre elles sont susceptibles d'intervenir simultanément lors du développement de fissures de CSC, ce qui rend l'analyse des signaux d'EA souvent délicate. 


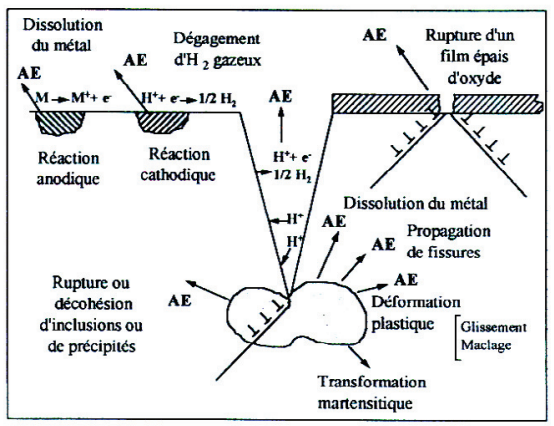

Figure 5. Sources potentielles d'EA associées à la propagation de fissures de corrosion sous contrainte [12].

De nombreux autres modes de corrosion localisée, eux-aussi détectables par EA (corrosion par piqûres, corrosion caverneuse...) sont également susceptibles de se développer simultanément avec la CSC, voire d'en être des moteurs d'amorçage, ce qui complique encore l'analyse, mais qui contribue aussi à la richesse de la technique : c'est en enregistrant des signaux d'amplitude moyenne et de faible fréquence, comparées aux caractéristiques des signaux de CSC enregistrés sur des aciers inoxydables sollicités en milieu chloruré chaud, que Proust a pu détecter un phénomène de piqûration concomitant à la CSC [13].

Par ailleurs, de la détection de processus annexes à la corrosion elle-même peut résulter une limitation importante dans la quantification des vitesses d'amorçage et/ou de propagation de l'endommagement. Il faut s'assurer de la corrélation des cinétiques d'endommagement avec les phénomènes émissifs, dont les sources «annexes » aux phénomènes de corrosion sont par ailleurs souvent peu énergétiques (exceptés les cas où une fissuration est impliquée). Leur détection par EA en sera d'autant plus influencée par la propagation des ondes au sein de la structure et par leur amortissement, et donc très sensible à la qualité de la chaîne de détection et de mesure utilisée.

\subsubsection{Détection de l'émission acoustique}

Avant d'être enregistrés à la surface de l'échantillon, les signaux d'émission acoustique subissent de multiples altérations durant leur propagation au sein de la structure (réflexion, réfraction, absorption, atténuation...). La transformation des ondes mécaniques arrivant en surface d'un matériau, en signaux d'émission acoustique, est réalisée par l'utilisation de capteurs piézoélectriques. Ceux-ci sont placés sur la surface du matériau, le couplage avec celui-ci étant assuré le plus souvent par l'utilisation d'un gel silicone. Le rôle du couplant est d'améliorer la transmission des ondes entre la surface de l'échantillon et le capteur.

A la sortie du capteur, le signal ne présente pas les qualités nécessaires à son transport jusqu'à la chaîne d'analyse. En effet, l'amplitude du signal délivré par un capteur piézoélectrique est de l'ordre du microvolt, en sortie haute impédance. Il est donc nécessaire de réaliser une adaptation d'impédance. C'est le rôle du préamplificateur.

\subsubsection{Apports de la technique dans le domaine de la fissuration assistée par l'environnement}

Une revue complète des applications de l'EA à la détection de fissures de CSC a été réalisée par Rothéa et Mazille il y a une quinzaine d'années [11]. Le lecteur pourra s'y référer pour avoir une vue d'ensemble des travaux réalisés jusqu'alors, classés selon les familles d'alliages étudiés. Bien évidemment, de nombreux travaux ont été entrepris depuis (71 références listées par le moteur de recherche INSPEC avec la requête « acoustic emission+stress corrosion cracking » depuis 1992); nous avons choisi d'illustrer notre propos au travers de 2 exemples issus de recherches récentes. 
3.2.2.1 Détection précoce de la fissuration interne par l'hydrogène [14] : Ce cas est intéressant à présenter car il permet d'illustrer 2 aspects spécifiques de la mise en œuvre de l'EA : l'intervention simultanée de différentes sources d'EA qu'il faut discriminer et la détection d'un endommagement interne. Les essais ont été conduits sur 2 nuances d'acier à microstructure ferrito-perlitique en milieu acide hydrogénant contenant de $l^{\prime} \mathrm{H}_{2} \mathrm{~S}$; la première nuance, qualifiée de «sour service», présente une microstructure homogène insensible à la fissuration HIC (décohésion interne sans contrainte appliquée) à $\mathrm{pH} \geq 4,5$ sous 1 bar d' $\mathrm{H}_{2} \mathrm{~S}$. La seconde nuance, qualifiée de «sweet service », présente une microstructure ferrito-perlitique avec des bandes de perlite, et est sensible à la fissuration de type HIC pour des $\mathrm{pH}<5,5$ sous 1 bar d' $\mathrm{H}_{2} \mathrm{~S}$. La procédure du test standardisé NACE a été adaptée de manière à maîtriser et suivre tous les paramètres expérimentaux et à assurer un suivi par émission acoustique [15].

Les sources d'EA actives pendant ce type d'essai, ainsi que les essais mis en œuvre pour les discriminer sont rassemblés dans le tableau 1.

Tableau 1. Sources potentielles d'EA en fonction des conditions d'essai et du matériau testé [14].

\begin{tabular}{|c|c|c|c||c|c|c|}
\hline \multicolumn{4}{|c||}{ Conditions expérimentales } & \multicolumn{3}{c|}{ Sources potentielles d'EA } \\
\hline milieu, remarque & gaz & $\mathrm{pH}$ & $\begin{array}{c}\text { matériau : } \\
\text { acier X65 }\end{array}$ & $\begin{array}{c}\text { Dégagement } \\
\mathrm{H}_{2} \text { (pop A) }\end{array}$ & $\begin{array}{c}\text { Présence } \\
\text { couche de } \\
\text { FeS (pop B) }\end{array}$ & $\begin{array}{c}\text { Fissuration } \\
\text { HIC (pop C) }\end{array}$ \\
\hline $\begin{array}{c}\mathrm{NaCl}, \mathrm{CH}_{3} \mathrm{COOH} \\
\text { polarisation } \\
\text { cathodique }\end{array}$ & 1 bar $\mathrm{N}_{2}$ & 5,5 & Sour Service & $\mathrm{X}$ & & \\
\hline $\mathrm{NaCl}, \mathrm{CH}_{3} \mathrm{COOH}$ & 1 bar $\mathrm{H}_{2} \mathrm{~S}$ & 4,5 & Sour Service & $\mathrm{X}$ & $\mathrm{X}$ & \\
\hline $\mathrm{NaCl}, \mathrm{CH}_{3} \mathrm{COOH}$ & 1 bar $\mathrm{H}_{2} \mathrm{~S}$ & 4,5 & Sweet Service & $\mathrm{X}$ & $\mathrm{X}$ & $\mathrm{X}$ \\
\hline
\end{tabular}

Cette démarche a permis l'identification de la signature acoustique de chaque source active (fig. 6), en particulier des signaux acoustiques liés à la fissuration de type HIC. L'enregistrement en cours d'essai des signaux liés à la fissuration permet ainsi la détection précoce de la fissuration de type HIC dans l'éprouvette. Les signaux enregistrés fournissent des informations sur les cinétiques des différents phénomènes émissifs ; il est ainsi possible de savoir quand la fissuration s'amorce. Nous constatons que l'énergie acoustique cumulée de la population associée à la couche de $\mathrm{FeS}$ croît rapidement en début d'essai (fig. 7). Un parallèle peut être fait entre cette évolution et les observations visuelles en cours d'essai qui ont permis de constater que la couche de FeS se formait en surface de l'éprouvette pendant les premières heures de l'essai. Les signaux enregistrés en début d'essai seraient donc essentiellement liés à la formation de la couche de FeS. L'évolution de la population de signaux associée au dégagement d'hydrogène est relativement constante au cours du temps, comme

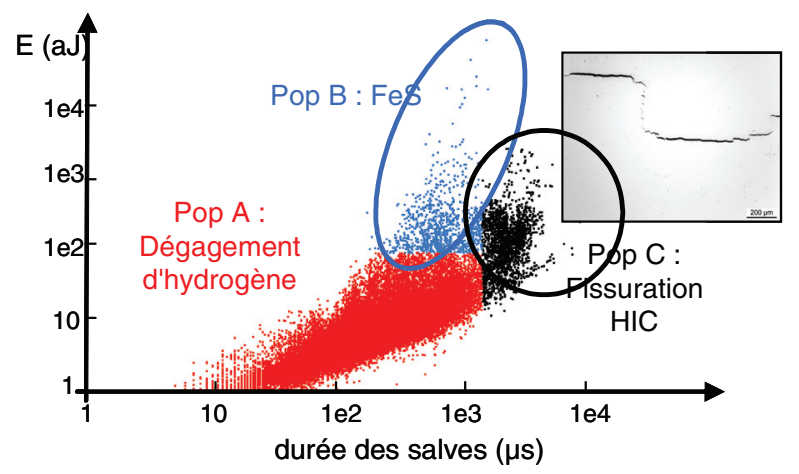

Figure 6. Corrélation de l'énergie absolue des salves d'EA en fonction de leur durée $\left(\mathrm{pH} 4.5,1\right.$ bar $\mathrm{H}_{2} \mathrm{~S}$, acier X65) [14]. 


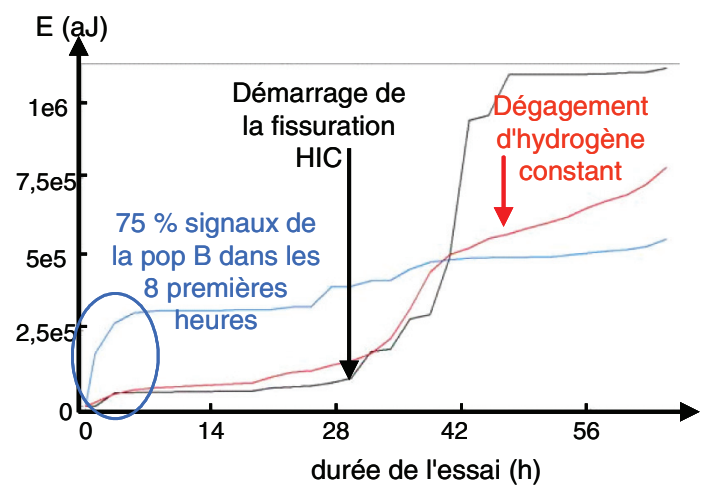

Figure 7. Évolution de l'énergie absolue cumulée (aJ) des différentes populations d'EA (durée de l'essai en heures, $\mathrm{pH}$ 4.5, 1 bar $\mathrm{H}_{2} \mathrm{~S}$, acier X65) [14].

le phénomène physique de dégagement d'hydrogène. L'énergie cumulée des signaux d'EA associés à la fissuration de type HIC augmente significativement après 28 heures d'essai. Le retard de 'démarrage' de la population associée à la fissuration de type HIC pourrait s'expliquer par le temps nécessaire à l'hydrogène pour diffuser, être piégé et se recombiner sous forme gazeuse, 'retardant' ainsi le début de la propagation de la fissuration de type HIC.

Ces résultats laissent augurer de la possibilité de corréler l'émission acoustique à l'amplitude de l'endommagement HIC et d'évaluer ainsi la sensibilité HIC de divers aciers uniquement grâce aux données acquises par la technique d'émission acoustique.

3.2.2.2 Détermination du mode de propagation des fissures : Dans de nombreux cas de CSC, la propagation des fissures prend un caractère mixte inter ou transgranulaire, qui correspond à des stades biens définis de leur développement. Il apparaît donc intéressant d'être informé en cours d'essai du mode de propagation des fissures. Prenons l'exemple de la CSC par l'iode des alliages de zirconium qui fissurent tout d'abord de manière intergranulaire, puis voient les fissures se propager de manière transgranulaire par quasi-clivage et fluting (fig. 2) au-delà d'un facteur d'intensité de contrainte critique. Des essais de CSC réalisés sur éprouvettes pré-écrouies ou non, permettent de faire varier la proportion de fissuration transgranulaire observée sur le faciès de rupture [16], la fissuration transgranulaire étant accompagnée d'une augmentation de l'émission acoustique enregistrée au cours de l'essai (fig. 8). Il apparaît donc que le début de fissuration transgranulaire puisse être détecté par EA. Deux mécanismes sources peuvent être à l'origine de cette détection : le quasi-clivage, induisant une libération d'énergie brutale au sein du matériau, mais également le phénomène de fluting résultant de l'accommodation par glissement prismatique des déformations imposées ; les mouvements de dislocations, s'ils sont rapides et coopératifs, sont en effet sources d'EA [17].

\subsection{Le suivi électrique (ou «Direct Current Potential Drop»)}

Cette technique nécessite l'utilisation d'éprouvettes pré-entaillées ou pré-fissurées ; elle ne permet donc d'obtenir que des informations relatives à la phase de propagation des fissures. Le principe de la technique est relativement simple : il consiste à appliquer un courant continu de forte intensité dans une éprouvette conductrice de géométrie bien déterminée et à mesurer la différence de potentiel aux bornes de la fissure à l'aide de deux électrodes (un courant alternatif peut aussi être appliqué, et on parle alors de «Alternative Current Potential Drop »). Lorsque la fissure se propage, l'augmentation de la surface fissurée induit une augmentation de la différence de potentiel. Moyennant un étalonnage préalable, l'évolution de ce potentiel en fonction du temps permet de suivre l'augmentation de la 

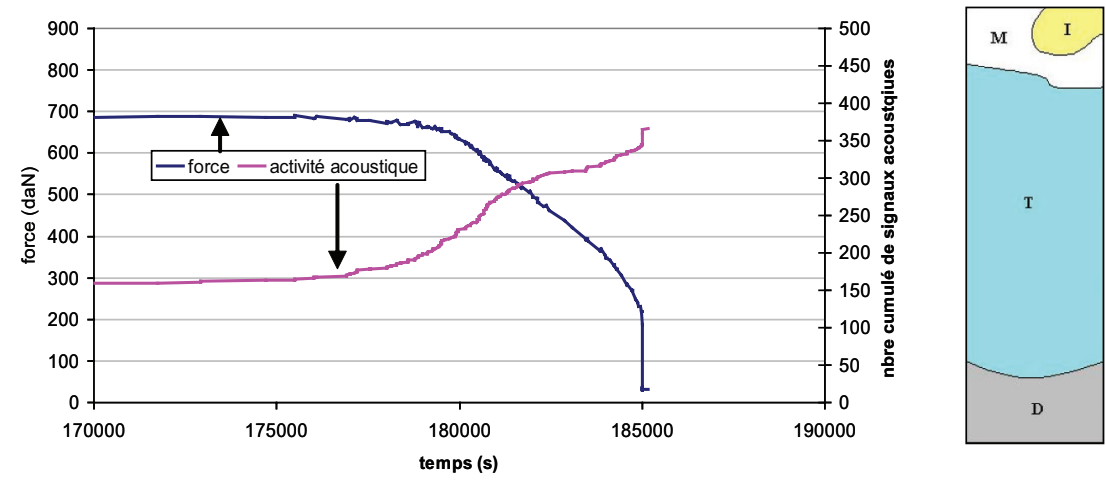

Figure 8. Évolutions conjointes de la charge appliquée et de l'activité acoustique lors de la propagation de fissures transgranulaires [16] (Zircaloy-4, méthanol iodé, $25^{\circ} \mathrm{C}$, I : intergranulaire, T : transgranulaire, D : ductile, $\mathrm{M}$ : mixte $\mathrm{T}+\mathrm{I})$.

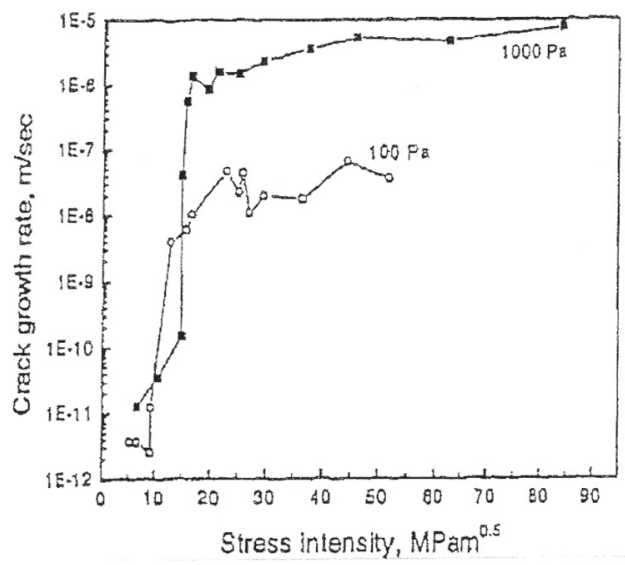

Figure 9. Influence de la pression partielle d'iode sur la vitesse de fissuration mesurée par DCPD (Zircaloy-2, iode vapeur, $350^{\circ} \mathrm{C}$ ) [18].

longueur de fissure. Pour les essais menés à haute température, une mesure de référence est utilisée pour pallier les variations de température qui induisent de fausses indications de variation de longueur de fissure (la résistivité du métal dépend de la température). La résistivité du métal dépend aussi de son taux de déformation ; pour une analyse quantitative, il faut donc prendre garde à la plasticité en pointe de fissure.

Cette technique est largement utilisée dans le domaine de la corrosion sous contrainte et de la fatigue corrosion. On peut l'illustrer par le cas de la CSC des alliages de zirconium par l'iode précédemment cité. Ghosal a utilisé le suivi électrique pour étudier l'influence de l'état métallurgique de l'alliage, de la température et de la pression partielle d'iode sur la sensibilité à la CSC [18]. Des mesures de la vitesse de propagation de fissures obtenue par DCPD ont été tracées en fonction de la valeur du facteur d'intensité de contrainte en fond de fissure recalculée au cours de l'essai grâce aux mesures DCPD (fig. 9). Les auteurs ont ainsi pu mettre en évidence que la valeur du facteur d'intensité de contrainte critique pour la propagation de fissures de CSC était indépendante de la composition de l'alliage et de son traitement thermique, qu'un plateau de vitesse de propagation en fonction du $\mathrm{K}_{\mathrm{I}}$ était observé pour les alliages recristallisés et qu'une augmentation de la température et de la pression partielle d'iode conduisait à une augmentation des cinétiques de fissuration. 
Dans ce cas particulier d'étude, la fissure se propageait exclusivement de manière transgranulaire par quasi-clivage et fluting. Mais nous avons vu que, pour ce couple matériau/milieu particulier, en fonction de la texture de l'alliage, la fissuration peut en premier lieu être intergranulaire, puis devenir transgranulaire. Les cinétiques de fissuration inter et transgranulaire peuvent être relativement différentes car les mécanismes physiques à leur origine ne sont pas les mêmes. Il apparait donc très intéressant de pouvoir avoir accès aux informations morphologiques et cinétiques simultanément. Le couplage de la technique d'émission acoustique et du suivi électrique peut permettre d'atteindre cet objectif. En cela, ces deux techniques revêtent des caractères tout à fait complémentaires.

\subsection{La microtomographie $\mathrm{X}$}

La microtomographie synchrotron est une technique non-destructive de caractérisation tridimensionnelle fournissant une cartographie 3D de $\mu$, le coefficient d'atténuation linéaire du matériau étudié. En chacun des voxels (élément de volume, par analogie avec pixel, élément d'image en 2D), la valeur de $\mu$ est la moyenne des coefficients d'atténuation des différents fluides et solides présents au moment de l'acquisition. Lorsque l'échantillon considéré est composé de matériaux, de phases ou de zones d'endommagement (comme c'est le cas d'une fissure) ayant des valeurs de $\mu$ très différentes, il est possible, par segmentation, de transformer la cartographie de $\mu$ en une image 3D de distribution des différentes zones de l'échantillon. De plus, la microtomographie étant non destructive, il est possible de modifier l'échantillon entre deux acquisitions et de suivre les évolutions induites en 3D avec une précision de l'ordre du micron. Ainsi, les précisions offertes par les synchrotrons de 3ème génération (actuellement à l'ESRF -European Synchrotron Radiation Facility, Grenoble- la résolution va de $0,3 \mu \mathrm{m}$ à $40 \mu \mathrm{m}$.) ouvrent des perspectives immenses pour la caractérisation et la modélisation des matériaux, en particulier dans le domaine de la corrosion sous contrainte.

Cet outil a par exemple été utilisé pour des observations in-situ de l'amorçage et de la propagation de fissures intergranulaires sur un acier inoxydable type 302 sensibilisé sollicité sous faible charge en milieu $\mathrm{K}_{2} \mathrm{~S}_{4} \mathrm{O}_{6}$ acide [19]. Les fissures sont amorcées par couplage de l'échantillon à une électrode de platine durant quelques secondes. La fissuration se développe alors à potentiel libre et peut être stoppée pour les observations tomographiques par un couplage à une électrode d'aluminium pur avec réduction simultanée de la charge appliquée. La propagation redémarre dès que l'éprouvette est découplée et la charge ré-augmentée. L'amorçage, la croissance et la coalescence des fissures menant à la rupture de l'échantillon ont ainsi pu être visualisés tout au long de l'essai après reconstruction de l'échantillon en 3 dimensions (fig. 10a) ou sur des coupes 2D (fig. 10b). Les mécanismes à l'origine du pontage entre fissures sont désormais étudiés et modélisés à partir des observations tomographiques [20].

\section{MÉTHODES INDUSTRIELLES DE DÉTECTION ET DE QUANTIFICATION DE L'ENDOMMAGEMENT PAR CSC}

Le coût lié à la détérioration d'installations industrielles par Corrosion Sous Contrainte constitue une part importante des coûts liés à la corrosion, dans l'industrie chimique notamment. C'est le mode de corrosion le plus insidieux, dans le sens où il conduit à des ruptures brutales, souvent imprévisibles. Dans la pratique industrielle, la détection de fissures de CSC permet de limiter leurs conséquences. Cette détection est rendue possible par l'utilisation de méthodes de contrôles non destructifs (CND). Les méthodes classiquement utilisées en corrosion, ou plus spécifiquement dans le domaine de la CSC sont décrites par la littérature [11] [21]. Le lecteur pourra s'y référer pour une description détaillée de leur principe. Le tableau 2 en présente un résumé. Nous nous proposons de discuter ici de leurs limites d'utilisation.

Pour la détection de la corrosion localisée sur site industriel, l'examen visuel (par un inspecteur certifié) des structures constitue bien souvent un guide de prise de décision : choix d'une méthode 
a
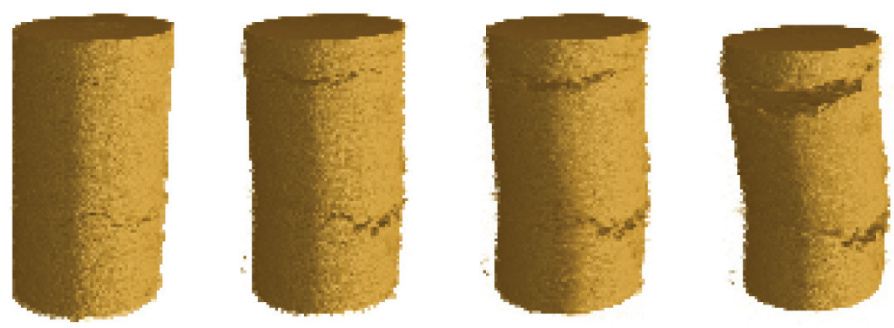

$\mathrm{b}$

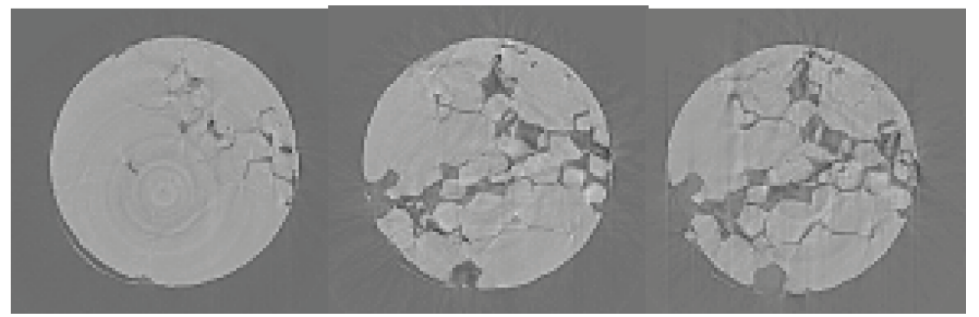

Figure 10. Visualisation en 3 dimensions (a) et en coupe (b) de la propagation de fissures intergranulaires par microtomographie $\mathrm{X}$ (rayonnement synchrotron, acier type 302 sensibilisé, $\mathrm{K}_{2} \mathrm{~S}_{4} \mathrm{O}_{6}$ acide) [19].

d'investigation plus poussée, arrêt de production... Cependant, du fait de leur finesse en surface, la détection visuelle des fissures de corrosion sous contrainte reste délicate et apparaît souvent trop tardive pour une approche prédictive.

Le contrôle par ressuage est souvent entrepris après examen visuel laissant suspecter l'existence de fissures de CSC. Le ressuage fluorescent est nettement plus performant que l'utilisation de lumière blanche, mais est plus difficile à mettre en œuvre sur site industriel. De ce fait, la sensibilité de la détection est abaissée et le repérage de fissures de CSC fines, comme c'est le cas pour les aciers inoxydables austénitiques sollicités en milieu chloruré, reste délicat.

Dans ce dernier cas particulier, très souvent rencontré dans l'industrie chimique, la sensibilité des techniques de radiographie et de magnétoscopie est également trop faible pour autoriser une approche préventive. Une autre limitation de la magnétoscopie réside dans le caractère non ferromagnétique des aciers austénitiques. Cette technique sera plus facilement applicable pour la détection de fissures de CSC sur acier au carbone, car elles sont en général plus ouvertes (mais dans ce cas, l'examen visuel suffit souvent !).

La mise en œuvre des ultrasons est également limitée par la nécessité d'une bonne propagation des ondes et donc par le caractère absorbant des structures (phase austénitique, présence de brides). Quant à la technologie TOFDT récemment développée [21] (exploitation des signaux ultrasonores diffractés par les bords des discontinuités présentes au sein de la pièce contrôlée), elle n'est applicable que pour des structures de forte épaisseur.

Ces limitations mises à part, toutes ces techniques ne permettent en général qu'un examen périodique des structures. Or, la phase d'amorçage des fissures de CSC peut être relativement lente, alors que la phase de propagation est en général très rapide. Le développement de techniques permettant un suivi des installations en continu apparait donc particulièrement intéressant. Parmi les techniques précédemment citées, l'émission acoustique offre une telle perspective. Elle permet en outre un contrôle volumique à $100 \%$ de la structure par un maillage adapté des capteurs, avec une très grande sensibilité de détection des défauts évolutifs en service, tout en autorisant une localisation de ces défauts. 
Tableau 2. Méthodes de contrôle non destructif classique utilisées pour la détection de la CSC sur site industriel.

\begin{tabular}{|c|c|}
\hline \multicolumn{2}{|r|}{ Examen visuel } \\
\hline Outil & CEil, endoscope, caméra robotisée ... \\
\hline Détection & Absence ou présence de corrosion, défaut de revêtement... \\
\hline Informations & Qualitatives \\
\hline Champ d'investigations & Plusieurs centaines de mètres de canalisations avec un endoscope robotisé \\
\hline Mise en œuvre & Contrôle à l'arrêt ou en service (dans ce cas, pas d'examen interne de la pièce) \\
\hline \multicolumn{2}{|r|}{ Contrôle d'étanchéité } \\
\hline Outil & Gaz traceur + spectromètre de masse \\
\hline Détection & Fuites dues à des fissures débouchantes \\
\hline Informations & Quantification difficile \\
\hline Champ d'investigations & Réservoirs, réacteurs, échangeurs \\
\hline Mise en œuvre & Contrôle à l'arrêt \\
\hline \multicolumn{2}{|r|}{ Ressuage } \\
\hline Outil & Liquide contenant un traceur coloré ou fluorescent, révélateur, observation visuelle \\
\hline Détection & Fissures débouchantes, non obstruées \\
\hline Informations & $\begin{array}{l}\text { Amplification de la largeur (sensibilité } 10 \mu \mathrm{m} \text { ) des fissures, bonne évaluation } \\
\text { du volume (sensibilité } 0.5 \mathrm{~mm} \text { ) }\end{array}$ \\
\hline Mise en œuvre & Contrôle à l'arrêt, problème d'accessibilité, préparation de surface préalable \\
\hline \multicolumn{2}{|r|}{ Magnétoscopie } \\
\hline Outil & $\begin{array}{l}\text { Aimantation de la pièce, révélateur dont les particules magnétiques s'accumulent } \\
\text { autour des défauts, observation visuelle }\end{array}$ \\
\hline Détection & Fissures débouchantes ou sub-surfacique $(<3 \mathrm{~mm})$ \\
\hline Champ d'investigations & Longueur des fissures $>0.5 \mathrm{~mm}$, largeur $>1 \mathrm{~mm}$, taille des défauts amplifiée \\
\hline Mise en œuvre & $\begin{array}{l}\text { Contrôle difficile pour grandes pièces et formes complexes, } \\
\text { matériaux ferromagnétiques uniquement }\end{array}$ \\
\hline \multicolumn{2}{|r|}{ Radiographie X et $\gamma$} \\
\hline Outil & Source rayons $\mathrm{X}$ ou g, observation visuelle des films \\
\hline Détection & Fissures dans le volume de la pièce \\
\hline Information & $\begin{array}{l}\text { Dépend de l'orientation des fissures par rapport à la direction principale } \\
\text { du rayonnement émis par la source, et de leur ouverture }\end{array}$ \\
\hline Champ d'investigations & $\begin{array}{l}\text { Pièces d'épaisseur } 10 \text { à } 100 \mathrm{~mm} \text { pour les } \mathrm{RX}, 40 \text { à } 150 \mathrm{~mm} \text { d'épaisseur } \\
\text { pour les rayons g }\end{array}$ \\
\hline Mise en œuvre & $\begin{array}{l}\text { Inspection périodique ou suivi en service de pièces ou structures en fabrication ou } \\
\text { maintenance }\end{array}$ \\
\hline \multicolumn{2}{|r|}{ Ultrasons } \\
\hline Outil & Transducteur émetteur-récepteur piézoélectrique \\
\hline Détection & Localisation des fissures \\
\hline Information & $\begin{array}{l}\text { Sensibilité dépend de l'orientation de la surface du défaut par rapport à la direction } \\
\text { principale du faisceau acoustique }\end{array}$ \\
\hline Champ d'investigations & $\begin{array}{l}\text { Ondes guidées : distance d'inspection dépend de la nature d'un éventuel revêtement } \\
\text { et du fluide transporté, propagation des ondes stoppée par des brides }\end{array}$ \\
\hline Mise en œuvre & $\begin{array}{l}\text { Inspections périodiques ou suivi en service d'installations avec ou sans } \\
\text { accès interne }\end{array}$ \\
\hline \multicolumn{2}{|r|}{ Courants de Foucault } \\
\hline Outil & $\begin{array}{l}\text { Bobine créant des courants induits par un flux magnétique variable, mesure de la } \\
\text { modification de l'impédance électrique de l'ensemble bobine-pièce }\end{array}$ \\
\hline Détection & $\begin{array}{l}\text { Fissures débouchantes ou sub-surfaciques, dimensions et orientation } \\
\text { des fissures (amplitude du signal), nature et profondeur (phase) }\end{array}$ \\
\hline Champ d'investigations & $\begin{array}{l}\text { Tubes, barres, feuillards, échangeurs de chaleur... } \\
\text { Sensibilité : longueur } 0.2 \mathrm{~mm} \text {, largeur } 1 \mathrm{~mm}\end{array}$ \\
\hline Mise en œuvre & $\begin{array}{l}\text { Inspections périodiques ou suivi en service d'installations, pour tout matériau } \\
\text { conducteur, sans nécessité d'accès interne si l'épaisseur est faible }\end{array}$ \\
\hline
\end{tabular}


Les deux grandes applications de l'EA dans l'industrie chimique sont ainsi :

- la caractérisation de l'intégrité d'une structure ; l'EA révèle en une seule opération les défauts évolutifs lors de variations de contrainte provoquées par une épreuve hydraulique (test en eau à $150 \%$ de la pression de calcul), un essai pneumatique ou en service ; lors du cycle de pression, les défauts évolutifs émettent des signaux d'émission acoustique qui sont recueillis par les capteurs permettant ainsi la localisation des défauts et leur classement selon une échelle de sévérité (qui ne permet cependant pas d'identifier la nature de ces défauts) ;

- l'écoute, sans variation de contrainte de procédé, des endommagements électrochimiques actifs (CSC, piqûres...) et donc l'étude de l'impact d'une modification de procédé (température, $\mathrm{pH} . .$. ) sur la tenue des matériaux ; on parle alors de «monitoring procédés ».

Dans le cas du contrôle des équipements sous pression, la technique d'EA constitue une alternative efficace à la ré-épreuve hydraulique et ses contrôles associés (nécessitant la vidange et la décontamination des structures et induisant une introduction d'eau et d'oxygène) et à la visite intérieure, préconisées pour leur requalification.

\section{CONCLUSIONS}

L'objectif de ce cours était de recenser les principales méthodes de détection et de quantification de l'endommagement par CSC, utilisées actuellement en laboratoire et sur site industriel. Nous avons vu que ces méthodes étaient très variées : métallographiques, fractographiques, électriques, acoustiques, électrochimiques, tomographiques.

Elles fournissent des informations sur la détection de l'amorçage des fissures, leur localisation, leur taille, leur vitesse de propagation, les mécanismes à l'origine de leur développement. Leur caractère très complémentaire confère au couplage de ces techniques entre elles des potentialités très intéressantes pour progresser dans la quantification in situ de l'endommagement. C'est dans cette voie que sont menées les études actuelles visant à atteindre ce but.

\section{Remerciements}

L'auteur tient à remercier L. Renaud (Arkéma) et A. Proust (EPA) pour les informations fournies concernant les méthodes de détection de la CSC sur site industriel, ainsi que B. Normand (INSA-Lyon) et C. Duret (Correx) pour leur relecture attentive et constructive.

\section{Références}

[1] Peyrat C., Raquet O., Helie M., Santarini G., Les Annales de Chimie - Science des Matériaux 24 (1999) 281.

[2] Raquet O., Feron D., Santarini G., in Advances in Mechanical Behaviour, Plasticity and Damage, Proceedings of Euromat 2000, edited by Miannay D., Coasta P., Françoise D. and Pineau A. (Elsevier Science Kidlington UK, 2000) pp. 1195-1200.

[3] Santarini G., Corrosion 455, (1989) 369.

[4] Paret A., Magnin T., Les Annales de Chimie - Science des Matériaux 24 (1999) 253.

[5] Herms E., Les Annales de Chimie - Science des Matériaux 24 (1999) 253.

[6] Fregonese M., Regnard C., Rouillon L., Magnin T., Lefebvre F., Lemaignan C., in Zirconium in the Nuclear Industry : $12^{\text {th }}$ International Symposium, edited by G.P. Sabol and G.D. Moan (ASTM-STP 1354, 2000) 377.

[7] Oltra R., in Corrosion Sous Contrainte : phénoménologie et mécanismes, Bombannes 1990, edited by Desjardins D. and Oltra R., (Les Editions de Physique, 1992) 49.

[8] Cottis R.A., Al-Ansari M.A., Bagley G., Pettiti A., Materials Science Forum, 289-292 (1998) 741. 
[9] Anita T., Pujar M.G., Shaikh H., Dayal R.K., Khatak H.S., Corrosion Science 48 (2006) 2689.

[10] Bosch R-W., Corrosion Science 47 (2005) 125.

[11] Rothéa R., Mazille H., in Corrosion Sous Contrainte : phénoménologie et mécanismes, Bombannes 1990, edited by Desjardins D.and Oltra R., (Les Editions de Physique, 1992) pp. 515.

[12] Yuyama S., ASTM Special Tech. Publ. 908 (1986) 75.

[13] Proust A., Etude par Emission Acoustique de la Corrosion Sous Contrainte de trois nuances d'aciers inoxydables (austénitique, ferritique et austénoferritique) en milieu chloruré chaud, Thèse de doctorat $n^{\circ}$ 92ISAL0081, Institut National des Sciences Appliquées de Lyon, 1992

[14] Smanio-Renaud V., Fregonese M., Kittel J., Cassagne T., Ropital F., Normand B., in Proceedings of Eurocorr 2007 (Friburg, Germany, 2007)

[15] NACE International Standard Test Method NACE TM0177-96, Laboratory testing of metals for resistance to specifics forms of environmental cracking in $\mathrm{H}_{2} \mathrm{~S}$ environments, NACE (1996)

[16] Fregonese M., Olagnon C., Godin N., Hamel A., Douillard T., Journal of Nuclear Materials, to be published

[17] Heiple C.R., Carpenter S.H., Journal of Acoustic Emission 6 (1987) 177.

[18] Ghosal S.K., De P.K., in Proceedings of the International Conference on Advances in Materials and Materials Processing, edited by N. Chakraboti and U.K Chatterjee, (McGraw Hill, New Dehli, 2002) pp. 139-146

[19] Marrow J., Withers P.J., Buffiere J.Y., Newman R.C., Engelberg D., Babout L., Johnson G., in ESRF Experimental Report: ME577, 2003. available at: http://ftp.esrf.fr/pub/UserReports/ 25143_A.pdf

[20] Marrow T.J., Babout L., Jivkov A.P., Wood P., Engelberg D., Stevens N., Withers P.J., Newman R.C., Journal of Nuclear Materials, 352 (2006) 62

[21] Caron D., Rivenez J., Bruez B., Examens non destructifs pour la détection de la corrosion $I^{r e}$ partie: généralités, in Prévention et lutte contre la corrosion - Une approche scientifique et technique, edited by B. Normand, N. Pébère, C. Richard and M. Very (Presses Polytechniques et Universitaires Romandes, 2004) pp. 409-436 
